\title{
Nonideal effects on inelastic Compton scattering in a nonideal plasma
}

\author{
Y O U N G-D A E J U N G ${ }^{1,2}$ \\ ${ }^{1}$ Department of Physics, 0319, University of California, San Diego, 9500 Gilman Drive, \\ La Jolla, CA 92093-0319, USA \\ ${ }^{2}$ Permanent address: Department of Physics, Hanyang University, \\ Ansan, Kyunggi-Do 425-791, South Korea \\ (ydjung@physics.ucsd.edu; yjung@bohr.hanyang.ac.kr)
}

(Received 17 May 2001)

\begin{abstract}
Inelastic Compton scattering of photons by hydrogenic ions in a classical nonideal plasma is investigated. An effective pseudopotential model taking into account plasma screening and collective effects is applied to describe the interaction potential in a nonideal plasma. The screened atomic wave functions and energy eigenvalues for the ground and excited states of the hydrogenic ion in a classical nonideal plasma obtained by the Ritz variational and perturbational methods. The expression for the lowest-order transition matrix element is obtained by a twophoton process associated with terms quadratic in the vector potential $\mathbf{A}$. The inelastic Compton scattering cross-section horn the $1 s$ ground state to the $2 p$ excited state is obtained as a function of the incident photon energy, Debye length, and the non-ideality plasma parameter. It is found that the collective effect reduces the cross-section. The collective effect on the cross-section is decreased with increasing Debye length.
\end{abstract}

\section{Introduction}

Compton scattering (Bethe and Salpeter 1957; Gould 1965, 1972, 1979, 1984; Tucker 1975; Cohen-Tannoudji et al. 1992) of photons by free or bound atomic electrons can serve as a probe of the atomic environment, since the spectra of radiation emitted from laboratory and astrophysical plasmas provide information about plasma parameters. The scattering of photons on bound atomic electrons (Longair 1992) has been the subject of special attention in many areas of physics and astrophysics, since this process is quite sensitive to the details of atomic structure and the correlation effects between atomic electrons. When the incident photon energy is greater than the excitation threshold energy of the target atom, the final state of the target atom may be different from the initial state, and this process is called inelastic Compton scatterings. Inelastic Compton processes for atoms in a dense plasma are different from those for free atoms owing to the screening effects of the surrounding plasma electrons. The Debye-Hückel potential describes the properties of a low-density plasma, and corresponds to a pair correlation approximation. Compton scattering in a weakly coupled plasma has been investigated using the DebyeHückel interaction potential (Jung 2000). A plasma described by the Debye-Hückel model is called an ideal plasma, since the average energy of interaction between 
particles is small compared with the average kinetic energy of a particle. However, multiparticle correlation effects caused by simultaneous interaction of many particles should be taken into account with increasing plasma density. Then, it is necessary to take into account not only short-range collective effects but also longrange effects in the case of a plasma with a moderate density and temperature. In this case, the interaction potential cannot be described by the Debye-Hückel model because of the strong collective effects of nonideal particle interaction (Baimbetov et al. 1995; Arkhipov and Davletov 1998; Arkhipov et al. 2000). Thus, Compton scattering of electron photons by bound atomic electrons in a classical nonideal plasma will be different from that in an ideal plasma. The radiative decay from the excited state to the ground state in a plasma has been known to be a useful tool in the study of plasma parameters (Jung 1995). Thus, in this paper, we investigate the plasma screening and collective effects on inelastic Compton scattering for the $1 s \rightarrow 2 p$ transition of the hydrogenic ion target in a classical nonideal plasma in order to investigate plasma screening and collective effects on the lowest optically allowed excitation process. Here we neglect relativistic effects (Bethe and Salpeter 1957) for bound-state wave functions, since we restrict ourselves to hydrogenic wave functions with $Z \alpha \ll 1$, where $\alpha\left(=e^{2} / \hbar c \approx \frac{1}{137}\right)$ is the fine-structure constant. The $1 s$ and $2 p$ screened atomic wave functions and corresponding energy eigenvalues of the hydrogenic ion with nuclear charge $Z$ are given by the Ritz variational method (Jung 1993; Hecht 2000). The expression for the transition matrix element is obtained from the basic lowest-order two photon-perturbation Hamiltonian.

In Sec. 2, we discuss the differential cross-section and the transition matrix element for inelastic Compton scattering. In Sec. 3, we obtain the screened $1 s$ and $2 p$ atomic wave functions and corresponding energy eigenvalues in a classical nonideal plasma using the Ritz variational calculation. In Sec. 4, we derive the inelastic Compton scattering cross-section for $1 s \rightarrow 2 p_{0}$ excitation in a classical nonideal plasma. We also investigate plasma screening and collective effects on the inelastic scattering cross-section with changing Debye length and nonideality plasma parameter. The results show that the collective effect reduces the inelastic Compton scattering cross-section. The collective effect on the cross-section is found to decrease with increasing Debye length. Finally, conclusions are given in Sec. 5 .

\section{Differential inelastic Compton scattering cross-section}

We assume that $|i\rangle$ represents the ground state with the energy eigenvalue $E_{i}$ of an atom whose center of mass is fixed at the origin and $|f\rangle$ represents the excited state with the energy eigenvalue $E_{f}$. For inelastic Compton scattering, the initial and final states of the total system including the atom and radiation field are given by $\left|\phi_{i}\right\rangle=\mid i ; \mathbf{k}_{i} \hat{\boldsymbol{\epsilon}}_{i}$ and $\left|\phi_{f}\right\rangle=\left|f ; \mathbf{k}_{f} \hat{\boldsymbol{\epsilon}}_{f}\right\rangle$, where $\mathbf{k}_{i}$ and $\mathbf{k}_{f}$ are the initial and final photon wave vectors and $\hat{\boldsymbol{\epsilon}}_{i}$ and $\hat{\boldsymbol{\epsilon}}_{f}$ are the unit photon polarization vectors. Using the Fermi golden rule in the Coulomb gauge (Cohen-Tannoudji et al. 1992), the differential scattering cross-section for inelastic Compton scattering can be represented by

$$
d \sigma_{f i}=\frac{2 \pi}{\hbar c}\left|M_{f i}\right|^{2} \delta\left(E_{f}-E_{i}\right) \frac{d^{3} \mathbf{k}_{f}}{(2 \pi)^{3}},
$$

where $M_{f i}$ is the transition matrix element,

$$
M_{f i}=\left\langle f ; \mathbf{k}_{f} \hat{\boldsymbol{\epsilon}}_{f}\left|H_{I}\right| i ; \mathbf{k}_{i} \hat{\boldsymbol{\epsilon}}_{i}\right\rangle .
$$


Here $H_{I}\left(=e^{2} A^{2} / m c^{2}\right)$ is the lowest-order two photon-perturbation Hamiltonian, where $\mathbf{A}$ is the vector potential of the radiation field. For a weak radiation field, such a two-photon process is known to be associated with terms quadratic in $\mathbf{A}$. At high photon energies, the second-order term in $\mathbf{p} \cdot \mathbf{A}$ can be almost neglected and the leading $A^{2}$ term dominates Compton scattering, where $\mathbf{p}(=(\hbar / i) \boldsymbol{\nabla})$ is the momentum operator. However, for resonant Compton scattering, which occurs at photon energies close to the resonance energy, the term $\sum_{j} \mathbf{p} \cdot \mathbf{A}\left(E-E_{j}\right) \mathbf{p} \cdot \mathbf{A}$ term is dominant, where $E_{j}$ is the energy of the intermediate state.

After some algebra, the differential Compton scattering cross-section for inelastic scattering from an arbitrary initial state $|i\rangle$ to an arbitrary final state $|f\rangle$ is then given by

$$
\frac{d \sigma_{f i}}{d \Omega}=\frac{\omega_{f}}{\omega_{i}} r_{0}^{2}\left|\left(\hat{\mathbf{\epsilon}}_{i} \cdot \hat{\boldsymbol{\epsilon}}_{f}\right)\left\langle f\left|e^{i\left(\mathbf{k}_{i}-\mathbf{k}_{f}\right) \cdot \mathbf{r}}\right| i\right\rangle\right|^{2},
$$

where $d \Omega$ is the solid angle about the direction $\mathbf{k}_{f}, r_{0}\left(=e^{2} / m c^{2}\right)$ is the classical electron radius, and $\omega_{i}$ and $\omega_{f}$ are the initial and final photon energies, respectively. The expression (3) is valid for arbitrary initial and final states, including transitions of multiple electrons. In the next section, we shall discuss plasma screening and collective effects on the atomic wave functions and energy eigenvalues in a classical nonideal plasma.

\section{Screened wave functions and energy eigenvalues}

Baimbetov et al. (1995) obtained an integro-differential equation for the effective potential of the particle interaction, taking into account the simultaneous correlations of $N$ particles, on the basis of a sequential solution of the chain of Bogolyubov equations for the equilibrium distribution function of particles of a classical nonideal plasma; an analytical expression for the pseudopotential of the particle interaction in a nonideal plasma was also obtained by application of the spline approximation. Using the pseudopotential, taking into account plasma screening and collective effects, the interaction potential $V(r)$ between an electron and an ion with charge $Z$ a classical nonideal plasma can be obtained by

$$
V(r)=-\frac{Z e^{2}}{r} e^{-r / \Lambda} \frac{1+\frac{1}{2} \gamma f(r)}{1+c(\gamma)},
$$

where $r$ is the distance between the electron and the nucleus, $\Lambda$ is the Debye length, $f(r)=\frac{1}{5}\left(e^{-\sqrt{\gamma} r / \Lambda}-1\right)\left(1-e^{-2 r / \Lambda}\right)$, and $\gamma\left(\equiv e^{2} / \Lambda k T_{e}\right)$ is the nonideality plasma parameter, $c(\gamma) \approx 0.456 \gamma-0.108 \gamma^{2}$ is the correlation coefficient for different values of $\gamma$, and $T_{e}$ is the electron temperature. When $\gamma \rightarrow 0$, i.e., in a weakly nonideal plasma, the pseudopotential (4) goes over to the Debye-Hückel interaction potential: $V(r) \rightarrow\left(-Z e^{2} / r\right) e^{-r / \Lambda}$. When an atom is placed in a dense plasma, its wave functions and energy eigenvalues are different from those of a free atom, because the nucleus is shielded by the surrounding plasma. Using the pseudopotential potential (4), the radial Schrödinger equation for a hydrogenic ion with nuclear charge $Z$ in a classical nonideal plasma is given by

$$
\left[-\frac{\hbar^{2}}{2 m}\left(\frac{d^{2}}{d r^{2}}-\frac{l(l+1)}{r^{2}}\right)-\frac{Z e^{2}}{r} e^{-r / \Lambda} \frac{1+\frac{1}{2} \gamma f(r)}{1+c(\gamma)}\right] P_{n l}(r)=E_{n l} P_{n l}(r),
$$

where $P_{n l}(r)$ and $E_{n l}$ are the radial wave function and energy eigenvalue of the 
electron in the $n l$ th shell, respectively. Here we consider an analytical variational method to obtain the solutions for the $1 s$ ground and $2 p$ excited states from (5), since the simple analytical solutions are quite useful for calculating the transition matrix elements. Then, we set the normalized variational $1 s$ and $2 p$ atomic wave functions, respectively, as follows:

$$
\begin{gathered}
P_{1 s}(r)=2 \alpha_{1 s}^{-3 / 2} r e^{-r / \alpha_{1 s}}, \\
P_{2 p}(r)=\frac{1}{2 \sqrt{6}} \alpha_{2 p}^{-5 / 2} r^{2} e^{-r / 2 \alpha_{2 p}},
\end{gathered}
$$

where $\alpha_{1 s}$ and $\alpha_{2 p}$ are the $1 s$ and $2 p$ variation parameters. For a pure Coulomb potential $V_{C}(r)\left(=-Z e^{2} / r\right)$, i.e., vanishing plasma screening and collective effects $(\Lambda \rightarrow \infty, \gamma \rightarrow 0)$, the variation parameters $\alpha_{1 s}$ and $\alpha_{2 p}$ become the Bohr radius $a_{Z}\left(=\hbar^{2} / Z m e^{2}\right)$ of a hydrogenic ion with nuclear charge $Z$.

From (5) and (6), the expectation value of the ground-state energy is obtained as

$$
\begin{aligned}
\left\langle E_{1 s}\left(\alpha_{1 s}\right)\right\rangle= & \frac{\hbar^{2}}{2 m \alpha_{1 s}^{2}}-\frac{Z e^{2}}{\alpha_{1 s}[1+c(\gamma)]}\left\{\left(1+\frac{\alpha_{1 s}}{2 \Lambda}\right)^{-2}+\frac{\gamma}{10}\left[\left(1+\frac{\alpha_{1 s}(\sqrt{\gamma}+1)}{2 \Lambda}\right)^{-2}\right.\right. \\
& \left.\left.-\left(1+\frac{\alpha_{1 s}}{2 \Lambda}\right)^{-2}-\left(1+\frac{\left.\alpha_{1 s} \sqrt{\gamma}+3\right)}{2 \Lambda}\right)^{-2}+\left(1+\frac{3 \alpha_{1 s}}{2 \Lambda}\right)^{-2}\right]\right\}
\end{aligned}
$$

The solution for the parameter $\alpha_{1 s}$ can be obtained by minimization of $\left\langle E_{1 s}\left(\alpha_{1 s}\right)\right\rangle$, i.e., $\partial\left\langle E_{1 s}\left(\alpha_{1 s}\right)\right\rangle / \partial \alpha_{1 s}=0$, since $\alpha_{1 s}$ is the variation parameter. The calculation of the variation parameter, using the perturbation method for $\alpha_{1 s}<\Lambda$, leads to the expression

$$
\alpha_{1 s}=\frac{a_{Z}}{\eta_{1 s}}
$$

where $\eta_{1 s}$ represents the plasma screening and collective effects on the Bohr radius of the $1 s$ state in a classical nonideal plasma:

$\eta_{1 s} \approx A(\gamma)\left[1-\frac{3}{4}\left(\frac{a_{Z}}{A(\gamma) \Lambda}\right)^{2}\left(1-\frac{2}{5} \gamma^{3 / 2}\right)+\left(\frac{a_{Z}}{A(\gamma) \Lambda}\right)^{3}\left(1-\frac{12}{5} \gamma^{3 / 2}-\frac{3}{5} \gamma^{2}\right)\right]$,

and $A(\gamma) \equiv[1+c(\gamma)]^{-1}$. This correction to $a_{Z}$ is quite reliable for $\Lambda \geqslant 10 a_{Z}$ and $\gamma \leqslant 1$. Using (8) and (9), the energy eigenvalue of the $1 s$ ground state becomes

$$
E_{1 s}=-Z^{2} R y\left(1-\delta_{1 s}\right)
$$

where $R y\left(=m e^{4} / 2 \hbar^{2} \approx 13.6 \mathrm{eV}\right)$ is the Rydberg constant and $\delta_{1 s}$ represents the plasma screening and collective corrections to the ground-state energy:

$$
\begin{aligned}
\delta_{1 s}(\Lambda, \gamma) \approx & 1-A^{2}(\gamma)-2 A(\gamma)\left(\frac{a_{Z}}{\Lambda}\right)+\frac{3}{2}\left(\frac{a_{Z}}{\Lambda}\right)^{2}\left(1-\frac{2}{5} \gamma^{3 / 2}\right) \\
& -\frac{1}{A(\gamma)}\left(\frac{a_{Z}}{\Lambda}\right)^{3}\left(1-\frac{12}{5} \gamma^{3 / 2}-\frac{3}{5} \gamma^{2}\right) .
\end{aligned}
$$


The expectation value of the $2 p$ excited state energy is also given by (5) and (7):

$$
\begin{aligned}
\left\langle E_{2 p}\left(\alpha_{2 p}\right)\right\rangle= & \frac{\hbar^{2}}{8 m \alpha_{2 p}^{2}}-\frac{Z e^{2}}{4 \alpha_{2 p}[1+c(\gamma)]}\left\{\left(1+\frac{\alpha_{2 p}}{\Lambda}\right)^{-4}+\frac{\gamma}{10}\left[\left(1+\frac{\alpha_{2 p}(\sqrt{\gamma}+1)}{\Lambda}\right)^{-4}\right.\right. \\
& \left.\left.-\left(1+\frac{\alpha_{2 p}}{\Lambda}\right)^{-4}-\left(1+\frac{\left.\alpha_{2 p} \sqrt{\gamma}+3\right)}{\Lambda}\right)^{-4}+\left(1+\frac{3 \alpha_{2 p}}{\Lambda}\right)^{-4}\right]\right\}
\end{aligned}
$$

The variation parameter $\alpha_{2 p}$ can also be determined from the minimization condition $\partial\left\langle E_{2 p}\left(\alpha_{2 p}\right)\right\rangle / \partial \alpha_{2 p}=0$. For $\alpha_{2 p}<\Lambda$, the approximate solution for $\alpha_{2 p}$ is found to be

$$
\alpha_{2 p}=\frac{a_{Z}}{\eta_{2 p}}
$$

where $\eta_{2 p}$ represents the plasma screening and collective effects on the Bohr radius of the $2 p$ state in a classical nonideal plasma:

$\eta_{2 p} \approx A(\gamma)\left[1-10\left(\frac{a_{Z}}{A(\gamma) \Lambda}\right)^{2}\left(1-\frac{2}{5} \gamma^{3 / 2}\right)+40\left(\frac{a_{Z}}{A(\gamma) \Lambda}\right)^{3}\left(1-\frac{12}{5} \gamma^{3 / 2}-\frac{3}{5} \gamma^{2}\right)\right]$

This expression is also quite reliable for $\Lambda \geqslant 10 a_{z}$ and $\gamma \leqslant 1$. Then, the energy eigenvalue for the $2 p$ excited state becomes

$$
E_{2 p}=-Z^{2} R y\left(1-\delta_{2 p}\right),
$$

where $\delta_{2 p}$ represents the plasma screening and correlation corrections to the $2 p$ excited-state energy:

$$
\begin{aligned}
\delta_{2 p}(\Lambda, \gamma) \approx & 1-A^{2}(\gamma)-8 A(\gamma)\left(\frac{a_{Z}}{\Lambda}\right)+20\left(\frac{a_{Z}}{\Lambda}\right)^{2}\left(1-\frac{2}{5} \gamma^{3 / 2}\right) \\
& -\frac{40}{A(\gamma)}\left(\frac{a_{Z}}{\Lambda}\right)^{3}\left(1-\frac{12}{5} \gamma^{3 / 2}-\frac{3}{5} \gamma^{2}\right) .
\end{aligned}
$$

In the next section, we shall discuss plasma screening and collective effects on $1 s \rightarrow 2 p_{0}(m=0)$ inelastic Compton scattering.

\section{Inelastic Compton scattering cross-section}

For inelastic Compton scattering from the $1 s$ ground state to the $2 p$ excited state, the final photon frequency $\omega_{f}$ is given by

$$
\omega_{f}=\omega_{i}-\frac{E_{2 p}-E_{1 s}}{\hbar}=\omega_{i}-\frac{3}{4 \hbar} Z^{2} R y\left(1-\Delta_{2 p, 1 s}\right),
$$

where $\Delta_{2 p, 1 s}$ represents the plasma screening and correlation effects on the threshold energy:

$$
\Delta_{2 p, 1 s}\left(a_{A}, \gamma\right) \cong 1-A^{2}(\gamma)+\frac{14}{3} a_{A}^{2}\left(1-\frac{2}{5} \gamma^{3 / 2}\right)-12 \frac{a_{\Lambda}^{3}}{A(\gamma)}\left(1-\frac{12}{5} \gamma^{3 / 2}-\frac{3}{5} \gamma^{2}\right),
$$

and $a_{\Lambda}\left(\equiv a_{Z} / \Lambda\right)$ is the scaled reciprocal Debye length. After some straightforward manipulations, the transition matrix element for $1 s \rightarrow 2 p_{0}(m=0)$ excitation is 


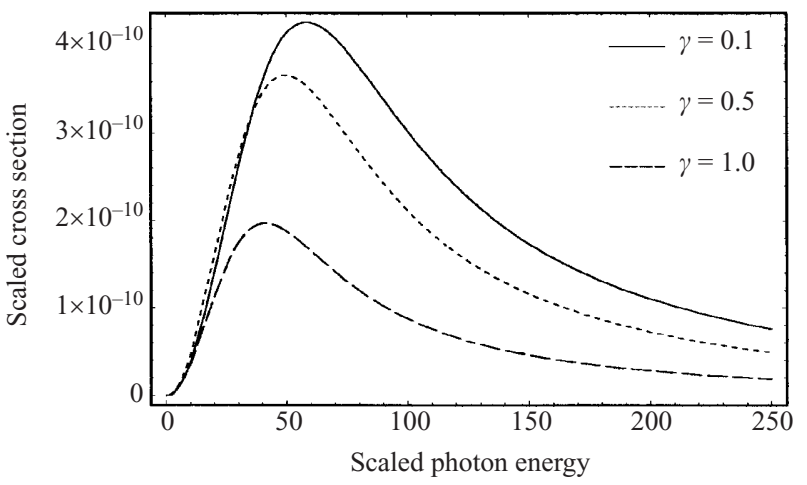

Figure 1. The $1 s \rightarrow 2 p_{0}$ inelastic Compton scattering cross-section $\sigma_{C}$ in units of $\pi a_{0}^{2}$ as a function of the scaled photon energy $\varepsilon_{i}\left(\equiv \hbar \omega_{i} / Z^{2} R y\right)$ for $Z=2$ and $a_{\Lambda}=0.1$.

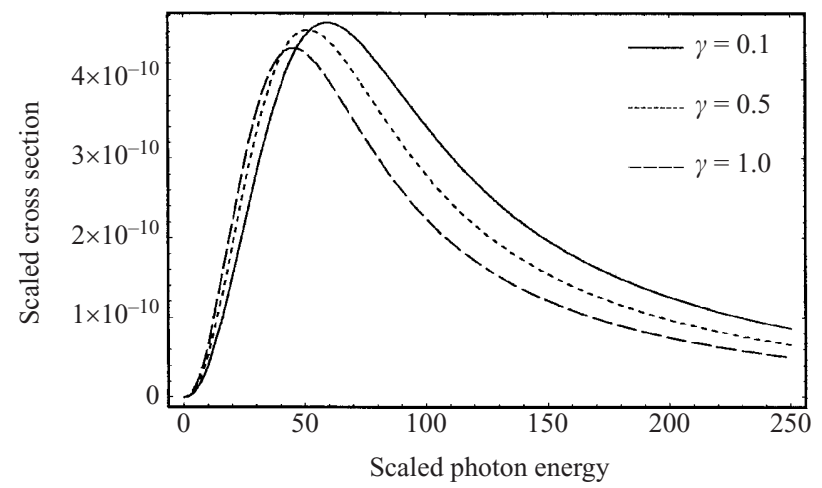

Figure 2. The $1 \mathrm{~s} \rightarrow 2 \mathrm{p}_{0}$ inelastic Compton scattering cross-section $\sigma_{C}$ in units of $\pi a_{0}^{2}$ as a function of the scaled photon energy $\varepsilon_{i}\left(\equiv \hbar \omega_{i} / Z^{2} R y\right)$ for $Z=2$ and $a_{\Lambda}=0.05$.

given by

$$
\left\langle 2 p_{0}\left|e^{i\left(\mathbf{k}_{i}-\mathbf{k}_{f}\right) \cdot \mathbf{r}}\right| 1 s\right\rangle=i 4 \sqrt{2} \eta_{1 s}^{3 / 2} \eta_{2 p}^{5 / 2} \frac{\left(\eta_{1 s}+\frac{1}{2} \eta_{2 p}\right)\left|\left(\mathbf{k}_{i}-\mathbf{k}_{f}\right) a_{Z}\right|}{\left[\left(\eta_{1 s}+\frac{1}{2} \eta_{2 p}\right)^{2}+\left|\left(\mathbf{k}_{i}-\mathbf{k}_{f}\right) a_{Z}\right|^{2}\right]^{3}} .
$$

By averaging the initial polarization, it follows that the expression for the inelastic Compton scattering cross-section $\sigma_{C}$ in units of $\pi a_{0}^{2}$ from the $1 s$ state to the $2 p_{0}$ state can be given as

$$
\begin{aligned}
\frac{\sigma_{C}}{\pi a_{0}^{2}}= & 2^{5} \eta_{1 s}^{3} \eta_{2 p}^{5}\left(\eta_{1 s}+\frac{1}{2} \eta_{2 p}\right)^{2} \alpha^{4}\left[1-\frac{3\left(1-\Delta_{2 p, 1 s}\right)}{4 \varepsilon_{i}}\right] \\
& \times \int_{0}^{\pi} d \theta \frac{\sin \theta\left(1+\cos ^{2} \theta\right)\left(\bar{k}_{i}^{2}+\bar{k}_{f}^{2}-2 \bar{k}_{i} \bar{k}_{f} \cos \theta\right)}{\left[\left(\eta_{1 s}+\frac{1}{2} \eta_{2 p}\right)^{2}+\bar{k}_{i}^{2}+\bar{k}_{f}^{2}-2 \bar{k}_{i} \bar{k}_{f} \cos \theta\right]^{6}},
\end{aligned}
$$

where $\varepsilon_{i}\left(\equiv \hbar \omega_{i} / Z^{2} R y\right)$ is the scaled incident photon energy, $\theta$ is the angle between $\mathbf{k}_{i}$ and $\mathbf{k}_{f}, \bar{k}_{i} \equiv k_{i} a_{Z}, \bar{k}_{f} \equiv k_{f} a_{Z}$, and the $1+\cos ^{2} \theta$ term is known as the angular phase for Thomson scattering (Hecht 2000).

In order to explicitly investigate the total plasma screening and collective effects on the inelastic Compton scattering cross-section from the $1 s$ ground state to the $2 p_{0}$ excited state, specifically, we consider three cases of the nonideal plasma parameter: 
Table 1. The numerical values of the $1 s \rightarrow 2 p_{0}$ inelastic Compton scattering cross-sections in units of $\pi a_{0}^{2}$ for $Z=2$ and $\varepsilon_{i}=100$.

\begin{tabular}{ccc}
\hline$\gamma$ & $\sigma_{C}\left(a_{\Lambda}=0.1\right) / \pi a_{0}^{2}$ & $\sigma_{C}\left(a_{\Lambda}=0.05\right) / \pi a_{0}^{2}$ \\
\hline 0.1 & $3.0270 \times 10^{-10}$ & $3.4135 \times 10^{-10}$ \\
0.5 & $2.1127 \times 10^{-10}$ & $2.7964 \times 10^{-10}$ \\
1.0 & $8.7087 \times 10^{-10}$ & $2.2419 \times 10^{-10}$ \\
\hline
\end{tabular}

$\gamma=0.1,0.5$, and 1 , and we assume that $Z=2$, since our nonrelativistic result $(21)$ is valid for $Z \alpha \ll 1$ because we ignore the relativistic effects. Figures 1 and 2 show the scaled inelastic Compton scattering cross-sections in units of $\pi a_{0}^{2}$ from the $1 s$ state to the $2 p_{0}$ state for $a_{\Lambda}=0.1$ and 0.05 , respectively. Numerical values of the inelastic Compton scattering cross-section in units of $\pi a_{0}^{2}$ are listed in Table 1 . As we can see from this table, the inelastic Compton scattering cross-section increases with decreasing reciprocal Debye length. It is also found that that the collective effect reduces the cross-section. The collective effect on the cross-section for $a_{\Lambda}=0.1$ is found to be more significant than that for $a_{\Lambda}=0.05$.

\section{Conclusions}

We have investigated the nonideal effect as well as the plasma screening effect on inelastic Compton scattering of photons by bound atomic electrons from the $1 \mathrm{~s}$ ground state to the $2 p$ state of hydrogenic ions in a classical nonideal plasma. The interaction potential in a classical nonideal plasma was obtained by the effective pseudopotential model, taking into account plasma screening and collective effects. The screened atomic wave functions and energy eigenvalues for the $1 s$ ground and $2 p$ excited states of the hydrogenic ion in a classical nonideal plasma were obtained by the Ritz variational and perturbational methods. The expression for the transition matrix element $M_{f i}$ was obtained from the lowest-order two photon-perturbation Hamiltonian $\left(H_{I} \propto A^{2}\right)$. It was found that the plasma screening effect reduces the inelastic Compton scattering cross-sections. It was also found that the collective effect reduces the cross-section. These results provide useful information on inelastic Compton scattering in a classical nonideal plasma.

\section{Acknowledgements}

The author is grateful to Professor R. J. Gould and Professor T. M. O’Neil for warm hospitality while visiting the University of California, San Diego.

This work was supported by the research fund of Hanyang University (HY-2001).

\section{References}

Arkhipov, Yu. V. and Davletov, A. E. 1998 Phys. Lett. 247A, 339.

Arkhipov, Yu. V., Baimbetov, F. B. and Davletov, A. E. 2000 Eur. Phys. J. D8, 299.

Baimbetov, F. B., Nurekenov, Kh. T. and Ramazanov, T. S. 1995 Phys. Lett. 202A, 211.

Bethe, H. A. and Salpeter, E. E. 1957 Quantum Mechanics of One-and Two-Electron Atoms. Berlin: Springer-Verlag.

Cohen-Tannoudji, C., Dupont-Roc, J. and Grynberg, G. 1989 Photons and Atoms. New York: Wiley.

Gould, R. J. 1965 Phys. Rev. Lett. 15, 577.

Gould, R. J. 1972 Ann. Phys. (NY) 69, 321. 
Gould, R. J. 1979 Astron. Astrophys. 76, 306

Gould, R. J. 1984 Astrophys. J. 285, 275.

Hecht, K. T. 2000 Quantum Mechanics. Berlin: Springer-Verlag.

Jung, Y. D. 1993 Phys. Fluids B5, 3432.

Jung, Y. D. 1995 Phys. Plasmas 2, 987.

Jung, Y. D. 2000 J. Plasma Phys. 64, 89.

Longair, M. S. 1992 High Energy Astrophysics, 2nd edn. Cambridge University Press.

Tucker, W. H. 1975 Radiation Processes in Astrophysics. Cambridge, MA: MIT Press. 